\section{Considering factors affecting the parental decision to abort after a prenatal diagnosis of a sex chromosome abnormality}

To the Editor: In their interesting study "Decision to Abort After a Prenatal Diagnosis of Sex Chromosome Abnormality: A Systematic Review of the Literature,"' Jeon and colleagues evaluated demographic factors to study associations with decisions to terminate or continue pregnancy. Although their review is a valuable contribution to the existing literature about the complex issue of decision-making after a prenatal diagnosis of a sex chromosomal aneuploidy (SCA), it does not address some relevant factors that might affect this decision-making. We would like to bring these factors to the readers' attention. Each of a multitude of factors, separately or in combination, may influence parental decisions about terminating pregnancy.

Various studies report that a fetal ultrasound abnormality strongly affects the decision to abort. ${ }^{2}$ The review reports indications for invasive prenatal testing in 12 of the 19 articles studied but does not address the reasons for referral. In our opinion, addressing these reasons is necessary. The authors report that young mothers ( $<36$ years old) are more likely to abort after being informed of a fetal SCA. However, as long as the reason for referral is unknown, the maternal age factor must be interpreted with great care. One must take into consideration that these young mothers may have been eligible for invasive prenatal testing on the indication of a fetal ultrasound abnormality. The prognosis is substantially poorer for a fetal ultrasound abnormality than for an incidental finding of SCA. The latter makes the decision-making even more burdensome because the phenotype may be quite normal but uncertainty remains concerning psychosocial development and late-onset diseases.

The important role of the Internet in providing anxious parents with extensive medical information is not mentioned in the review. We checked whether the informative role of the Internet was considered in the articles reviewed and discovered that it was not, even though the Internet has played an ever greater role in public information since the 1990s. Medical professionals must be aware of the fact that patients check the Internet for extensive information about their disease and/or possible treatments. Parents search the Internet looking for additional information about the syndromes for which their unborn children are diagnosed. In a qualitative interview study (as opposed to a quantitative one) that we published in $2011,{ }^{3}$ all the participating couples independently searched the Internet and stated that the information they found was a shock to them. After professional counseling, they decided that the Internet information was too negative, and ultimately they decided to continue pregnancy. We found two articles regarding the impact of medical information that patients can find on the Internet, ${ }^{4,5}$ and we believe that more research on this important issue will follow in the near future. In the complex of emotions after the unforeseen diagnosis of a fetal SCA, parents have a need for additional information; they start searching the Internet even before the posttest counseling appointment. The impact of searching the Internet for their decision-making is an important factor that we believe deserves more attention in future research.

Directive counseling is an important factor in the review by Jeon and colleagues. It is associated with the decision to terminate pregnancy, but the authors do not report the professional qualifications of the counselors or consider whether one type of counselor is more tempted to direct parents to abort than another. The profession of the counselor has been reported in 11 of the 19 studies, and the influence of the counselor's profession on the decision to terminate is elaborately discussed in 6 of these studies. Other publications report that counseling by nongeneticists may be associated with more decisions to terminate. ${ }^{6,7}$

The authors used a methodological quality score for the articles they reviewed. It would increase the usability of their review if each of the 19 articles in the table was accompanied with its individual score.

We conclude that (i) the reason for referral plays a major role in the process of decision-making when a fetal SCA is diagnosed, as fetal ultrasound abnormalities may influence the prognosis. The presence or absence of a fetal ultrasound abnormality was not addressed, and this fact affects the results of the review in important ways; (ii) the Internet has an increasingly important role in providing additional, but sometimes confusing, medical information to anxious parents who are searching unguided for help in their difficult decision-making; (iii) geneticists can make a significant contribution to the field of decision-making of parents to continue or to abort their pregnancy.

\section{DISCLOSURE}

The authors declare no conflict of interest.

Jacqueline J.P.M. Pieters, $M D^{1}$, Angelique J.A. Kooper, PhD', Ton B. Feuth, $\mathrm{MSc}^{3}$ and Arie P.T. Smits, PhD ${ }^{2}$

${ }^{1}$ Department of Obstetrics and Gynecology, Radboud University Nijmegen Medical Centre, Nijmegen, The Netherlands; ${ }^{2}$ Department of Human Genetics, Radboud University Nijmegen Medical Centre, Nijmegen, The Netherlands; ${ }^{3}$ Department of Epidemiology, Biostatistics and Health Technology Assessment, Radboud University Nijmegen Medical Centre, Nijmegen, The Netherlands.E-mail: j.pieters@obgyn.umcn.nl

\section{REFERENCES}

1. Jeon KC, Chen LS, Goodson P. Decision to abort after a prenatal diagnosis of sex chromosome abnormality: a systematic review of the literature. Genet Med 2012;14:27-38.

2. Mezei G, Papp C, Tóth-Pál E, Beke A, Papp Z. Factors influencing parental decision making in prenatal diagnosis of sex chromosome aneuploidy. Obstet Gynecol 2004;104:94-101.

3. Pieters JJ, Kooper AJ, Eggink AJ, et al. Parents' perspectives on the unforeseen finding of a fetal sex chromosomal aneuploidy. Prenat Diagn 2011;31:286-292. 
4. Lorence DP, Park H, Fox S. Assessing health consumerism on the Web: a demographic profile of information-seeking behaviors. J Med Syst 2006:30:251-258.

5. McMullan M. Patients using the Internet to obtain health information: how this affects the patient-health professional relationship. Patient Educ Couns 2006;63:24-28.

6. Hall S, Abramsky L, Marteau TM. Health professionals' report of information given to parents following the prenatal diagnosis of sex chromosome anomalies and outcomes of pregnancies; a pilot study. Prenat Diagn 2003;23:535-538.

7. Marteau TM, Nippert I, Hall S, et al.; DADA Study Group. Decision-making after diagnosis of fetal abnormality. Outcomes of pregnancies diagnosed with Klinefelter syndrome: the possible influence of health professionals. Prenat Diagn 2002;22:562-566.

doi:10.1038/gim.2012.32

\section{Response to Pieters et al.}

To the Editor: We wish to thank Pieters, Kooper, Smits, and Feuth for their interest in our review. We appreciate their thoughtful comments and the important factors raised in their letter "Considering Factors Affecting the Parental Decision to Abort After a Prenatal Diagnosis of a Sex Chromosome Abnor-

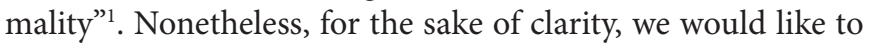
point out that our review ${ }^{2}$ extends beyond an analysis of demographic factors to include nondemographic variables such as type of sex chromosome abnormality and parents' fears.

No doubt the confounding factors raised by Pieters and colleagues (reason for referral, the role of the Internet, and qualifications of counseling providers) are extremely important for more fully understanding decisions to continue or terminate a sex chromosome abnormality-affected pregnancy. Unfortunately, these factors did not emerge as findings in the studies we reviewed, as they were not sufficiently explored in this body of work. Therefore, we thank Pieters and colleagues for helping to highlight and reinforce both this gap and our call for betterquality studies. To date, assessments of decisions surrounding a sex chromosome abnormality-affected pregnancy have been limited in their ability to identify the multiple factors shaping these decisions, the nuanced variability within those factors, and the complex interactions among them. Studies that (i) tap into the context of decision-making, (ii) examine how parents obtain information on their own through the Internet, and (iii) explore the role played by various types of service providers, in-depth-are urgently needed!

Moreover, as we argue in our review, studies of better methodological quality are also missing from this body of literature. Given the multifactor nature of the topic, it makes little sense to capture complex associations among factors and decisions using descriptive or bivariate statistical analyses without controlling for confounders or covariates. Also missing from this literature are qualitative studies that portray parents' points of view and voices, directly.

Finally, given the below-average methodological quality scores of most studies in the review, we chose not to report each study's score individually. These are available, however, upon request to the authors.

\section{DISCLOSURE}

The authors declare no conflict of interest.

Kwon Chan Jeon, MS ${ }^{1}$, Lei-Shih Chen, PhD, $\mathrm{CHES}^{1}$ and Patricia Goodson, $\mathrm{PhD}^{1}$

${ }^{1}$ Department of Health \& Kinesiology, Texas A\&M University, College Station, Texas, USA.E-mail:kc_jeon@hlkn.tamu.edu

\section{REFERENCES}

1. Pieters JJPM, Kooper AJA, Smits APT, Feuth TB. Considering factors affecting the parental decision to abort after a prenatal diagnosis of a sex chromosome abnormality. Genet Med 2012;14:558-559.

2. Jeon KC, Chen LS, Goodson P. Decision to abort after a prenatal diagnosis of sex chromosome abnormality: a systematic review of the literature. Genet Med 2012;14:27-38.

doi:10.1038/gim.2012.33 